\author{
Suzanne E. Anderson \\ Lynne S. Steinbach \\ Dechen Tschering-Vogel \\ Matthias Martin \\ Ladislav Nagy
}

\section{MR imaging of avascular scaphoid nonunion before and after vascularized bone grafting}

Received: 26 November 2004

Revised: 19 January 2005

Accepted: 25 January 2005

Published online: 15 April 2005

(C) ISS 2005

Paper presented at ESSR, European Society of Skeletal Radiology, Aarhus, Denmark, 14 June 2003. Awarded first prize for the ESSR award for Scientific Presentations Paper presented at ISS, Closed Scientific Session, Malta, 3 October 2004 as part of the above prize

S. E. Anderson (®) • D. Tschering-Vogel • M. Martin

Department of Radiology,

University Hospital of Bern, Inselspital, Freiburg Strasse, 3010 Bern, Switzerland e-mail: suzanne.anderson@insel.ch

Tel.: +41-31-6322435

Fax: +41-31-6324874

L. S. Steinbach

Department of Radiology,

University of California San Francisco,

San Francisco, California, USA

L. Nagy

Hand Surgery Division,

Department of Orthopedic Surgery,

University Hospital of Bern, Inselspital,

Bern, Switzerland

\begin{abstract}
Objective: To investigate the magnetic resonance (MR) imaging appearances of chronic nonunion of the scaphoid with proximal pole avascular necrosis before and after insertion of a vascularized bone graft, using computed tomography (CT) as the imaging gold standard. Design and patients: A retrospective study was performed involving MR imaging $(n=26)$, CT scans $(n=37)$ and radiographs $(n=52)$ of 13 men (mean age 29 years, age range 20-38 years) with avascular scaphoid nonunion. Avascular necrosis of the scaphoid proximal pole was confirmed intraoperatively $(n=13)$. MR images were acquired preoperatively and following placement of a vascularized bone graft. Scaphoid MR signal characteristics were assessed for evidence of vascular bone graft incorporation and revascularization of the bone marrow of the proximal pole of the scaphoid and compared with the gold standard of CT. Surgical and clinical notes were reviewed with a minimum 3 year imaging and clinical follow-up
\end{abstract}

in all patients. Results: Graft incorporation with revascularization of the proximal pole of the scaphoid was documented in 9 patients (69\%). Graft failure with persistent pseudoarthrosis and avascular necrosis of the scaphoid was seen in 4 patients (31\%). Conclusions: MR imaging is useful to determine whether vascularized bone graft incorporation and revascularization of the proximal pole of the scaphoid has occurred in the setting of avascular scaphoid nonunion.

Keywords Bone marrow, MR . Bones, avascular necrosis - Wrist, MR · Fracture

\section{Introduction}

Vascularized pedicle bone grafts $[1,2,3,4,5,6]$ offer significant advantages over conventional bone grafts in which healing occurs by creeping substitution on a dead bone matrix. They allow for healing that is similar to natural fracture healing though at a faster and more reliable rate [8], allowing for cell viability, replacement of deficient bone or osteogenesis and revascularization of ischemic bone [1, 2, 4, 5, 7]. This method has been used in treating Kienbock's disease (avascular necrosis (AVN) of the carpal lunate), fractures of the proximal pole of the scaphoid, and chronic nonunion of scaphoid fractures, including those with AVN of the proximal pole or those with failed prior surgery [4]. 
MR imaging is thought to be helpful in the evaluation for revascularization of vascularized bone grafts in the setting of Kienbock's disease [4]; however, to date there have been no MR imaging series regarding vascularized bone grafts for avascular scaphoid nonunion. CT has been used to determine graft union; however, the bone marrow findings within the proximal pole of the scaphoid may be appropriately assessed with MR imaging. We therefore present the MR imaging findings of 13 patients with chronic nonunion of the scaphoid with proximal pole AVN before and after vascularized bone graft.

\section{Materials and methods}

Patient informed consent was obtained prior to all imaging studies. Surgery was performed as part of accepted clinical patient care with accepted clinical indications. Institutional review board approval was not required.

Patients

Thirteen patients with avascular scaphoid nonunion were included in this retrospective MR imaging study. All patients were male, with a mean age of 29 years and an age range of 20-38 years. Inclusion criteria were the presence of avascular bone marrow appearance on T1- and T2-weighted imaging of the proximal pole of the scaphoid in the setting of chronic nonunion on the preoperative MR images and intraoperative confirmation of avascularity by inspection and the treatment with a vascularized bone graft. Patients with complicated class $2 \mathrm{~b}[1,2] \mathrm{AVN}$ of the proximal pole of the scaphoid (sclerosis with collapse) on radiography were excluded from the study as they did not receive vascularized bone grafts. A minimum of 3 years of clinical and imaging follow-up was available in all patients.

Imaging

Standard radiographs of the hand with posteroanterior and lateral views were performed. The pseudoarthrosis/nonunion was defined by the radiographs and MR imaging. Postoperative CT was performed using a Somatom Plus 4 scanner (Erlangen, Germany), at 3 months postoperatively and thereafter at 6-weekly intervals in the absence of consolidation. The CT slice thickness was $2 \mathrm{~mm}$, the table movement was $3 \mathrm{~mm}$ and the increment was $1 \mathrm{~mm}$ with reconstructions in both the axial and coronal planes. The CT scans were used as the imaging gold standard for incorporation and consolidation of the bone graft and to review for the presence or absence of the pseudoarthrosis. MR imaging was used to evaluate for the presence of AVN within the proximal pole of the scaphoid prior to surgery.

Each patient underwent preoperative and postoperative MR imaging performed on a $1.5 \mathrm{~T}$ superconducting magnet (Signa; GE Medical Systems, Milwaukee, Wis.). T1-weighted images were acquired in the coronal and axial plane with a repetition time (ms)/ echo time (ms) of 400-600/17, a matrix size of $512 \times 256$, NEX 4 and FOV 10, with $3 \mathrm{~mm}$ slice thickness and an interslice gap of $1 \mathrm{~mm}$. T2-weighted MR images were acquired in the axial plane by using the fast SE technique, a repetition time (ms)/echo time (ms) of 3400-6000/85-102, a matrix size of $256 \times 256$, NEX 4, FOV 10 and slice thickness of $3 \mathrm{~mm}$ with an interslice gap of $1 \mathrm{~mm}$. Short tau inversion recovery (STIR) images were obtained with a repetition time (ms)/echo time (ms)/inversion time (ms) of 3540/17/
150, a matrix size of $256 \times 192$, NEX 4, $3 \mathrm{~mm}$ slice thickness and $0 \mathrm{~mm}$ interslice gap. All patients received intravenous gadopentetate dimeglumine (Magnevist; Schering, Berlin, Germany) at a dose of $0.1 \mathrm{mmol} / \mathrm{kg}$ body weight. Fat-suppressed T1-weighted images $(n=26)$ following contrast enhancement were acquired in both the coronal and axial planes. Fat suppression was accomplished by using selective presaturation of lipid resonant frequency.

\section{Image evaluation}

Twenty-six MR images, 52 radiographs and $37 \mathrm{CT}$ scans of the 13 patients were retrospectively reviewed by two radiologists, with review by consensus. The CT scans and MR images were reviewed separately during different review sessions.

All patients had documented chronic pseudoarthrosis of the scaphoid. Presence of sclerosis, morphology of the proximal pole of the scaphoid, presence of nonunion and graft incorporation were reviewed on radiographs. MR signal characteristics of the proximal and distal pole of the scaphoid bone marrow were reviewed before and after surgery as well as the appearances of the bone marrow after contrast administration. The presence or absence of bone graft incorporation and osseous union of the pseudoarthrosis was documented with postoperative CT scans using axial images of the scaphoid [9] with reformatting performed in both the coronal and sagittal planes. True union was defined when there was evidence of continuity and uninterrupted trabeculae between the former scaphoid pseudoarthrosis on more than three contiguous sections. Pseudoarthrosis was defined as a breach of the trabecular bone, separating the scaphoid into a proximal and a distal portion.

Postoperative MR imaging was used to assess the revascularization of proximal pole of the scaphoid. Failures of revascularization were defined as those with persistent postoperative pseudoarthrosis formation with AVN of proximal pole of the scaphoid. Positive revascularization of the proximal pole of scaphoid was defined as normalizing bone marrow signal intensity on all MR imaging sequences with incorporation of the graft and an absence of the pseudoarthrosis. Normalizing of the bone marrow signal within the proximal pole of the scaphoid was defined as the same or very similar signal intensity within the marrow compared with the signal of the bone marrow in other adjacent carpal bones. Additionally the contrast enhancement within the proximal pole of scaphoid paralleled that of the other carpal bones in successful revascularization. Pseudoarthrosis was defined as a persistence of the breach in the trabecular bone of the scaphoid, with persistent division of the scaphoid into two parts. Status of the scapholunate ligament from MR imaging was also documented.

\section{Surgical procedure}

AVN of the proximal pole of scaphoid was confirmed intraoperatively with inspection of scaphoid bone surfaces. If the proximal pole osseous surface remained pale after debridement with no surface bleeding, and no bleeding after removal of the surgical tourniquet, a diagnosis of AVN of the proximal pole of the scaphoid was made. Eight patients were operated on by one of the two staff members with experience in general hand surgical techniques, the other five by hand fellows. The surgical technique used in all cases was that described by Zaidemberg et al. [3] (Fig. 1). A miniature Herbert screw or K-wires were used as the method of postoperative fixation. 

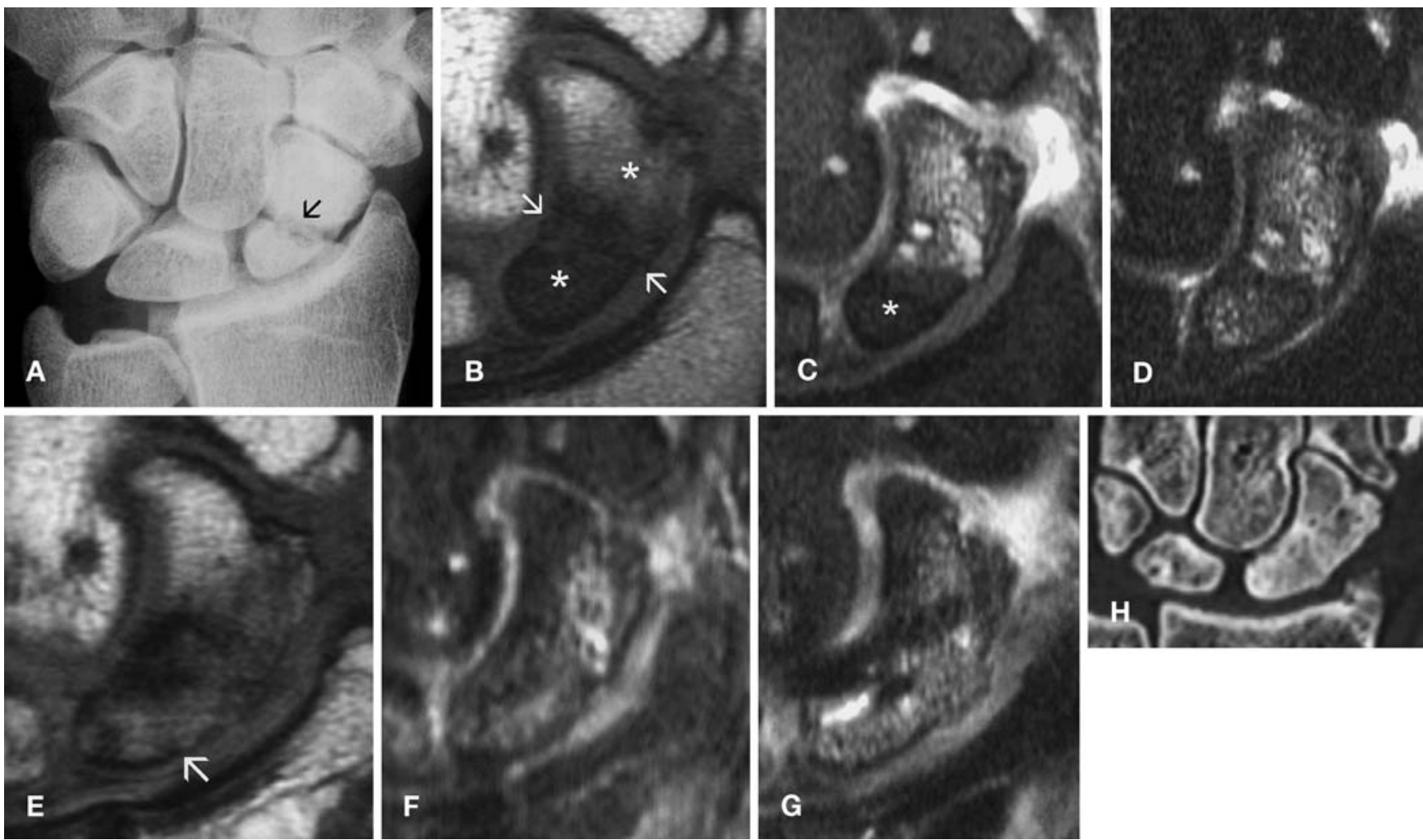

Fig. 1A-H A 29-year-old man with scaphoid nonunion and proximal pole avascular necrosis (AVN) with good clinical outcome following surgery. A Preoperative posteroanterior wrist radiograph demonstrates diffuse sclerosis within the proximal pole of the scaphoid and pseudoarthrosis (arrow). B Preoperative coronal T1-weighted MR image (TR: 460, TE: 16) shows decreased marrow signal intensity within the scaphoid proximal pole $(*)$ and additionally in the distal pole (*) with pseudoarthrosis (arrows). C Corresponding Short Tau Inversion Recovery (STIR) MR image (TI: 90, TR: 3800, TE: 19) shows decreased marrow signal intensity in the proximal pole $(*)$ and increased signal intensity within the distal pole of the scaphoid. D Fat-saturated T1-weighted MR image (TR: 600, TE: 17) after contrast enhancement shows in-

creased contrast enhancement within the proximal and distal pole of scaphoid. E Postoperative T1-weighted MR image (TR: 600, TE: 17) shows some evidence of normalizing marrow signal within the proximal pole (arrow) and evidence of union on MR imaging. Some persistent decreased signal intensity remains within both the proximal and distal poles, though this has markedly improved compared with the preoperative imaging. F STIR image (TI: 90, TR: 3840, TE: 19) shows increased signal intensity within the marrow of the proximal scaphoid and decreasing signal within the distal pole of the scaphoid. G Fat-saturated T1-weighted MR image (TE: 480, TE: 17) after contrast enhancement shows absence of pseudoarthrosis and evidence of consolidation. H Coronal reconstructed CT image confirms scaphoid consolidation
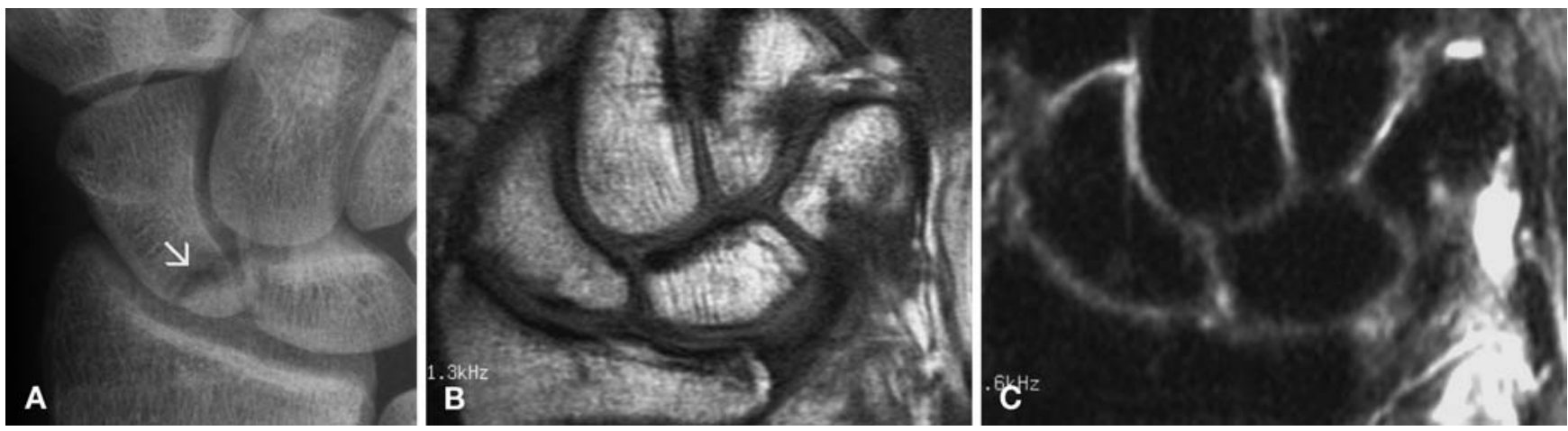

Fig. 2A-C Scaphoid nonunion with a proximal pole AVN which healed with surgery. A Preoperative radiograph of scaphoid nonunion (arrow) in a 20-year-old man with an avascular proximal pole on MR imaging confirmed with surgery. B Follow-up coronal T1-weighted MR image (TR: 600, TE: 17) 11 weeks after surgery shows consolidation of the bone graft and normalization of marrow signal within the proximal scaphoid. C Corresponding Short Tau Inversion Recovery image (TI: 90, TR: 3540, TE: 16) shows normalized marrow signal in the proximal scaphoid 


\section{Results}

Imaging results

Radiography showed evidence of subtle sclerosis within the proximal pole of the scaphoid in 7 patients (54\%) (Figs. 1, $2,3)$. There was no morphological alteration, such as collapse or fragmentation of the proximal pole of the scaphoid. Scaphoid pseudoarthroses were located in the proximal third to mid-third in all cases. MR signal characteristics are summarized in Table 1. On preoperative MR images there was decreased signal intensity on T1-weighting within the bone marrow of the proximal scaphoid in all patients. Notably there was a persistent decreased signal intensity within the bone marrow of the proximal pole of the scaphoid on T1-weighting in all 4 patients with failed surgical outcomes. At surgery, AVN of the proximal pole of the scaphoid was confirmed in all cases.
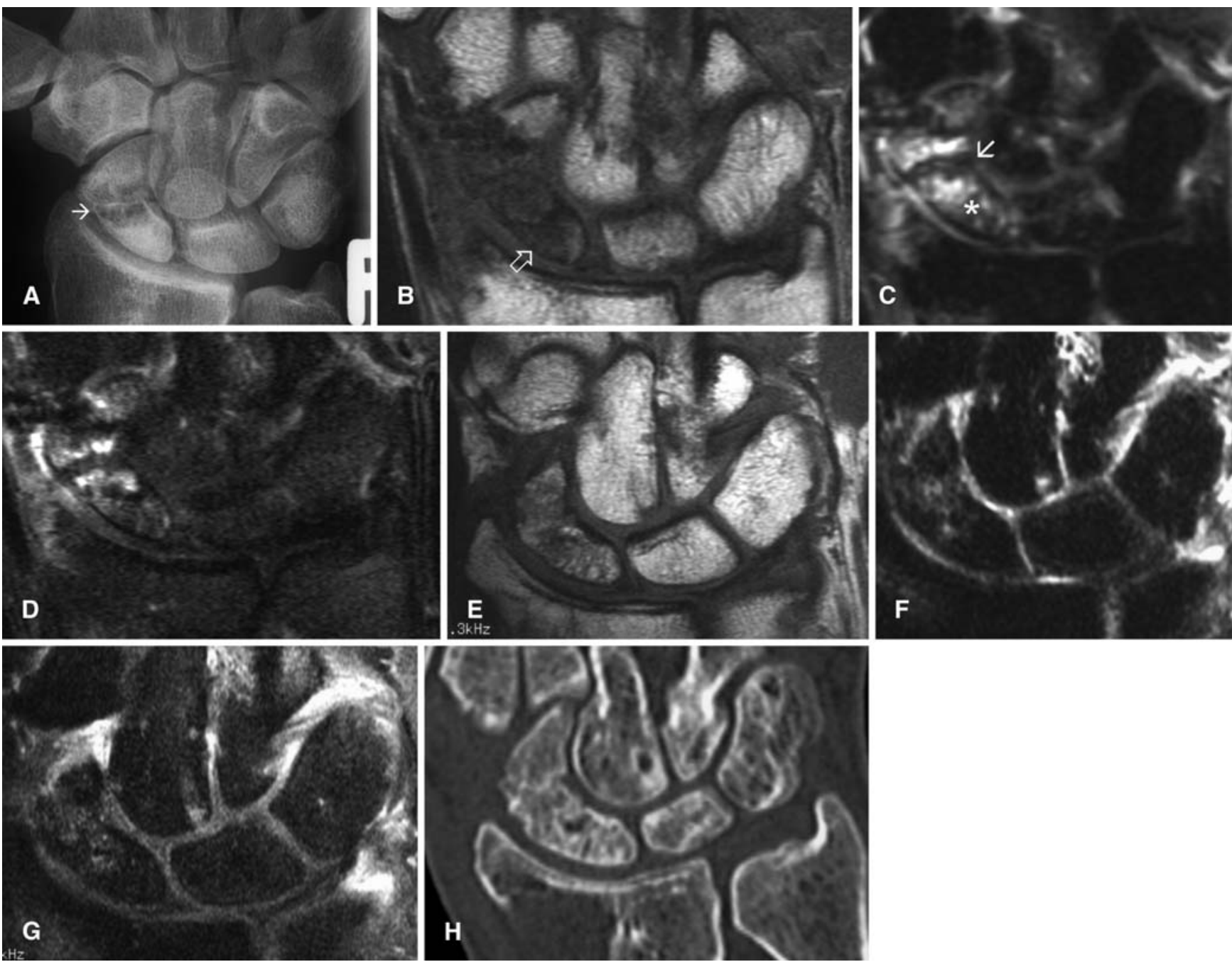

Fig. 3A-H A 28-year-old man with scaphoid nonunion and proximal pole AVN with good outcome following surgery. A Preoperative posteroanterior wrist radiograph demonstrates scaphoid nonunion (arrow) and sclerosis of the proximal pole. B Preoperative coronal T1-weighted MR image (TR: 532, TE: 20) shows decreased marrow signal within the proximal pole of the scaphoid (hollow arrow) and around the site of pseudoarthrosis. C Corresponding coronal Short Tau Inversion Recovery (STIR) image (TI: 90, TR: 3540, TE: 16) shows increased signal within the proximal pole of the scaphoid (*) and site of pseudoarthrosis (arrow). D Fatsaturated T1-weighted MR image (TR: 480, TE: 17) after contrast enhancement shows irregular contrast enhancement within the proximal pole of the scaphoid and around the site of pseudoarthrosis. E Follow-up coronal T1-weighted MR image (TR: 600, TE: 17) after surgery shows incorporation of the bone graft and normalizing marrow signal in the proximal pole of the scaphoid. $\mathbf{F}$ Corresponding coronal STIR image (TI: 90, TR: 3540, TE: 16) shows a normalizing of marrow signal within the scaphoid. G Fatsaturated T1-weighted MR image after contrast enhancement (TR: 480, TE: 16) shows a more normal marrow signal within the scaphoid, with contrast enhancement paralleling the bone marrow in adjacent carpal bones. H Follow-up coronal reconstructed CT image confirms consolidation of the scaphoid 

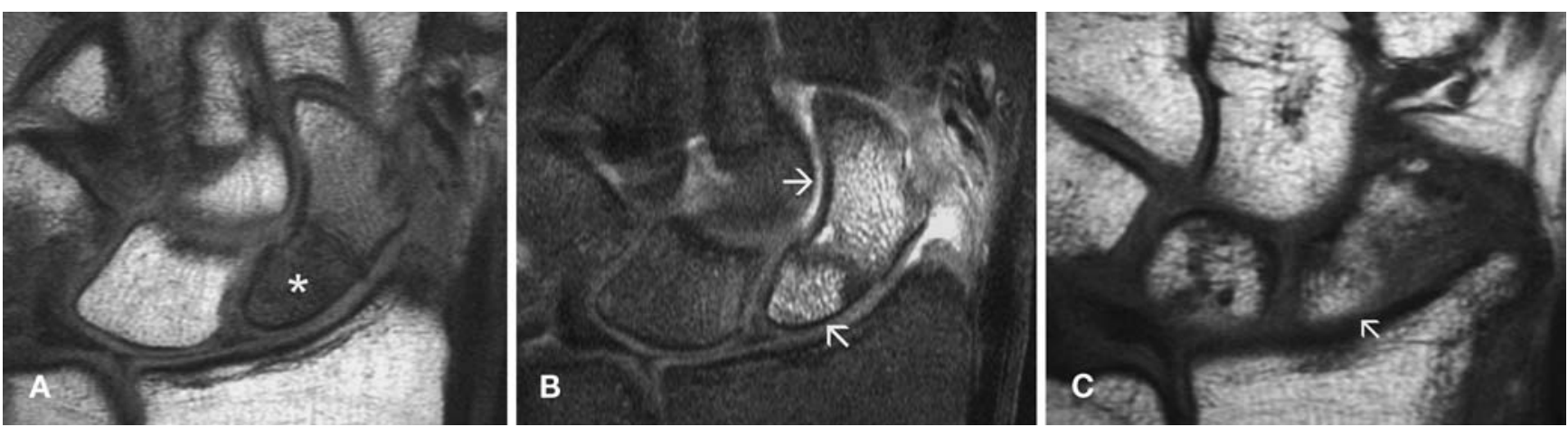

Fig. 4A-C Example of marked increased signal intensity within bone marrow after contrast administration in a patient with intraoperatively proven AVN of the proximal pole of the scaphoid. A Preoperative coronal T1-weighted MR image (TR: 600, TE: 17) with decreased signal intensity $(*)$ within the proximal pole of the scaphoid. B Fat-saturated T1-weighted MR image (TR: 480, TE: 16) after contrast enhancement shows a marked increase in signal

Table 1 Avascular scaphoid nonunion (+, radiographic sclerosis, avascular proximal pole at surgery, positive outcome; $\mathrm{N}$, normal bone marrow signal intensity compared with other carpal bones; $\downarrow$, decreased signal intensity; $\uparrow$, increased signal intensity; \, intensity (arrows) within both the proximal and distal scaphoid poles, despite absence of bleeding points intraoperatively. C Postoperative coronal T1-weighted MR image (TR: 600, TE: 17) shows a return of normal marrow signal intensity within the proximal pole of the scaphoid (arrow) after successful vascularized bone graft surgery predominantly decreased in signal intensity; $\nearrow$, predominantly increased in signal intensity; bold arrows indicate a marked bone marrow signal alteration; duration in days to consolidation of the pseudoarthrosis was defined by CT)

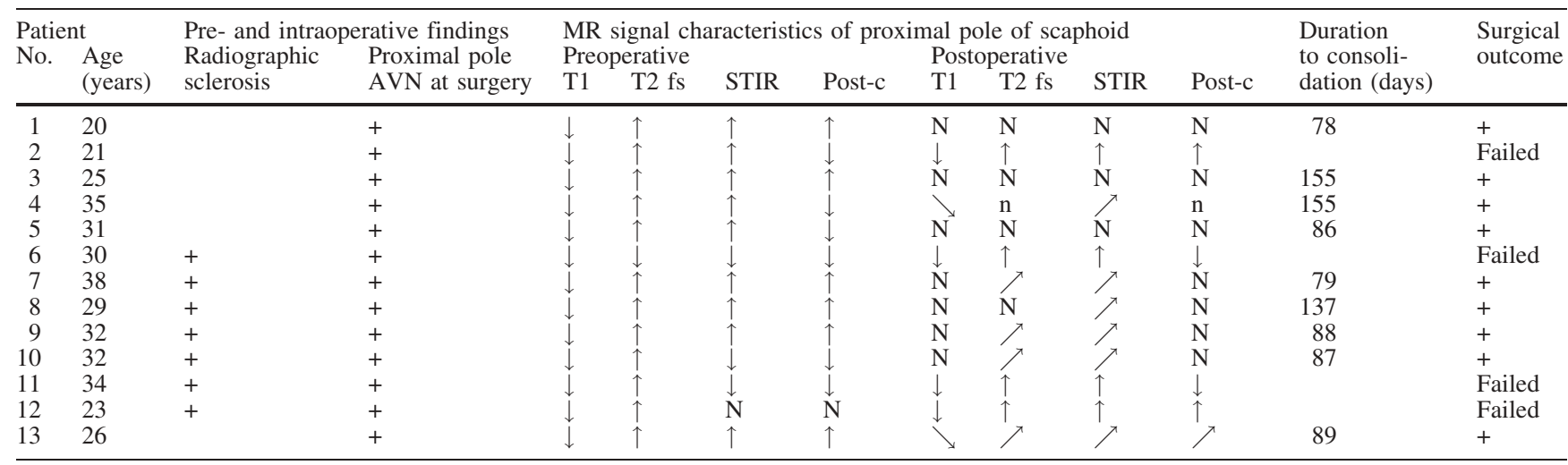

Consolidation and vascularized bone graft incorporation was evident on CT scans in the 9 patients on average at 106 days ( 3.5 months) with a range from 86 days to 155 days. In all 9 patients $(69 \%)$ there was evidence of revascularization (Figs. 2, 3, 4) of the proximal pole of scaphoid on the MR follow-up at 1.4 years on average (range $0.65-3.85$ years) with a normalization of the bone marrow signal within the proximal pole of the scaphoid on all MR imaging sequences compared with the signal intensity within the bone marrow of adjacent carpal bones. All 9 patients with successful outcomes had a normal-appearing scaphoid morphology. There was persistent pseudoarthrosis with persistent proximal pole AVN in 4 patients (31\%). Preoperative radiography, preand postoperative MR signal characteristics and surgical outcome are summarized in Table 1. Six patients, 3 of whom had more marked findings, had an elevated signal intensity in the proximal pole on T1-weighted fat-satu- rated images following intravenous contrast administration, with an avascular proximal pole at surgery. Scapholunate ligaments were intact in all 13 cases, possibly allowing for a theoretically intact alternative collateral vascular supply to the proximal pole of the scaphoid.

\section{Discussion}

Chronic nonunion of the scaphoid complicated by proximal pole AVN is variably reported in the orthopedic literature, the incidence ranging from $16 \%$ to $49 \%$ when an increase in the radiographic density is used as the defining criterion [1]. The presence of devascularization of bone within the proximal pole of the scaphoid at surgery, as inferred by the lack of bleeding points, has an incidence of $50-70 \%$ [2]. In clinical practice, the term 
AVN is used to label a wide spectrum of conditions of assumed circulatory compromise [2]. There is an overall union rate for scaphoid nonunion pseudoarthrosis of between $80 \%$ and $97 \%$ using long-term immobilization, internal fixation and conventional graft techniques [1,2]. Higher union rates are seen with vascularized bone graft techniques [1, 2]. Zaidemberg et al. [3] and Yuceturk et al. [7] achieved union with vascularized bone graft techniques in 11 and 4 cases respectively, with complete relief of chronic wrist pain. However, neither author commented on the vascularity of the nonunions treated. An experimental animal study showed $73 \%$ healing of the vascularized grafts and no healing of the conventional grafts in treatment of carpal fracture nonunions with AVN [8]. Graft failure was quite high in this series $(30.7 \%)$ compared with the literature, presumably due to the new technique involved.

Currently the literature suggests that the artery labeled as the 1,2 intercompartmental supraretinacular artery, a radial arterial branch, is preferred for pedicled vascularized grafts in scaphoid nonunion $[3,4,5,6]$. Zaidemberg et al. in 1991 [3] described this ascending irrigating branch, which has a pedicle with a short arc of rotation, with multiple small nutrient vessels, ideally suited for scaphoid grafts. The bone graft site is centered $1.5 \mathrm{~cm}$ proximal to the radiocarpal joint on the dorsal side. The scar tissue from both sides of the scaphoid nonunion is resected, leaving a structural defect of variable size. This is filled with the vascularized bone graft to maintain the anatomical shape of the scaphoid. There is a need to evaluate for bone healing in these cases as the interface between the avascular proximal pole of the scaphoid and the vascularized bone graft is at risk of failure of incorporation and revascularization, particularly if there is suboptimal contact of the surfaces or suboptimal fixation with movement.

To date there have been no series documenting the MR imaging appearances after vascularized bone grafting to the scaphoid. Shin et al. stated in 1998 that MR imaging showed revascularization in the setting of Kienbock's disease of the lunate [4]. Previous MR literature regarding the status of vascularity of the proximal pole of the scaphoid, has concentrated on MR signal characteristics classical for AVN, with decreased signal on T1-weighting and T-2 weighting compared with muscle signal [10]. In 1997 Schmidt et al. described three patterns of MR contrast enhancement [10]. In MR imaging stage I, representing bone marrow edema but not AVN, a homogeneous, sometimes excessive contrast enhancement was noted. In stage II, inhomogeneous enhancement correlated with coexisting AVN and areas of viable bone. In stage III, which represented AVN, there was no contrast enhancement. In 2000 Cerezal et al. advocated the use of preoperative contrast enhancement to quantify the extent of necrosis of the proximal pole, stating that lack of contrast strongly correlated with AVN and that contrast enhancement showed good correlation with surgical and histological findings with a positive vascular status of the proximal pole of the scaphoid and good healing potential of the pseudoarthrosis [11]. In our series, on preoperative images there was evidence of an increase in signal intensity after contrast administration in 6 patients, with a marked increase in signal intensity in 3 of these patients (Fig. 4) in the proximal pole of the scaphoid, which according to the current literature [11] should be evidence of bone marrow viability. However, this was not the case as these 3 cases were avascular with no evidence of punctate bleeding intraoperatively. The exact cause for this apparent increased contrast pattern remains unknown. In this series, in spite of variable preoperative MR signal intensities within the proximal pole of the scaphoid, attesting to the variable nature of altered or decreased bone vascularity, positive bone vascularity within healed bone fragments looked the same in all cases, with normalization of the MR bone marrow signal. The time period involved in the revascularization process was variable, as summarized in Table 1. As long as the scaphoid components had maintained their anatomical morphology there was a normalizing of the proximal pole bone marrow signal intensity on all MR imaging sequences (at least partial if not complete) after vascularized bone graft incorporation when compared with the bone marrow signal intensity of the adjacent carpal bones. Ganapathi et al. [12] described a return of normal marrow signal within the proximal pole of the scaphoid as a sign of union, with 6 of their 11 patients undergoing bone grafting, although they do not specify whether they used vascularized grafts.

With evidence of consolidation present in this series of patients between 3 and 4 months postoperatively with MR evidence of revascularization of the proximal pole of the scaphoid, we recommend this to be the optimal time for imaging. Scapholunate ligaments were intact and confirmed at surgery. This may possibly allow for intact collateral vascular supply to the proximal pole of the scaphoid [11] and therefore we recommend review for scapholunate ligament status on preoperative MR imaging.

Possible etiologies for graft failure in our series include the fact that the technique is new with a learning curve for the surgeon (three of the four surgical failures were performed by fellows with less experience), that the bone graft was avascular or poorly fitted, or that there was poor fixation. Other etiologies include incomplete postoperative immobilization and lack of patient compliance. In these 4 patients there was persistent abnormal MR signal intensities within the bone marrow of the proximal pole of the scaphoid in all sequences and persistent pseudoarthrosis as summarized in Table 1. MR imaging has the potential to influence clinical decision-making in the postoperative period, with an earlier diagnosis of graft failure and lack of proximal pole reperfusion that can possibly lead to early repeat surgery. 
One limitation of this study is its retrospective nature. All images had been discussed with the surgeon prior to operation so that he was not masked to the findings; however, all were clinical patients and it would have been unethical not to be informed of the MR results. Another limitation is the small number of selected patients included in the series and lack of control patients; however, vascularized bone graft surgery is only indicated in specific patients. All patients had AVN of the proximal pole of the scaphoid with normally maintained morphology. Seven of 13 patients had subtle sclerosis on radiography, which agrees with the findings of Sakuma et al. [13] who found no characteristic signal changes on MR imaging associated with radiographic sclerosis in the proximal pole of the scaphoid with nonunion using nonvascularized bone graft, supporting the lack of a relationship between sclerosis and AVN. All had proximal third to mid-third scaphoid pseudoarthrosis, which is more commonly associated with AVN and a poorer outcome [14]. Though the superselection of patients is a limitation of this study, vascularized bone graft surgery is recommended and thought optimal for this patient group [1, 2, 3, 4, 5]. Initially all patients had evidence on MR imaging consistent with AVN, but for inclusion into the study this had to be confirmed with intraoperative findings of AVN [13]. The preoperative and intraoperative diagnosis of AVN of the scaphoid may be difficult, as was discussed by Günal et al. in 1999 [15]. In this study the diagnoses matched in 19 patients but there was no correlation in 13 patients. Their recommendation was that the diagnosis of proximal pole of scaphoid AVN should only be made when both MR imaging and intraoperative findings indicate avascularity.

In conclusion, MR imaging is useful in the initial diagnosis of AVN and in monitoring the efficacy of surgical treatment in this patient population. MR imaging can determine whether vascularized bone graft incorporation and revascularization of the proximal pole of the scaphoid has occurred in the setting of avascular scaphoid nonunion. It can potentially influence decision-making by allowing early prognosis of graft healing.

\section{References}

1. Buechler U, Nagy L. Review article: The issue of vascularity in fractures and non-unions of the scaphoid. J Hand Surg [Br] 1995; 20:726-735.

2. Buechler U, Nagy L. To use or not to use vascularized bone grafts in treating nonunions of the scaphoid. Current Trends Hand Surg 1995; 89-95.

3. Zaidemberg C, Siebert JW, Angrigiani C. A new vascularized bone graft for scaphoid nonunion. J Hand Surg [Am] 1991; 16:474-478.

4. Shin AY, Bishop AT, Berger RA. Vascularized pedicled bone grafts for disorders of the carpus. Techn Hand Upper Extrem Surg 1998; 2:94-109.

5. Shin AY, Bishop AT. Pedicled vascularized bone grafts for disorders of the carpus: scaphoid nonunion and Kienbock's disease. J Am Acad Orthop Surg 2002; 10:210-216.
6. Sheetz KK, Bishop AT, Berger R. The arterial blood supply of the distal radius and its potential use in vascularized pedicled bone grafting. J Hand Surg [Am] 1995; 20:902-914.

7. Yuceturk A, Isiklar U, Tuncay C, Tandogan R. Treatment of scaphoid nonunions with a vascularized bone graft bases on the first dorsal metacarpal artery. J Hand Surg [Br] 1997; 22:425-427.

8. Sunagawa T, Bishop AT, Muramatsu K Role of conventional and vascularized bone grafts in scaphoid nonunion with avascular necrosis: a canine experimental study. J Hand Surg [Am] 2000; 25:849-859.

9. Sanders WE. Evaluation of the humpback scaphoid by computerized tomography in the longitudinal axial plane of the scaphoid. J Hand Surg [Am] 1988; 13:182-187.

10. Schmitt R, Heinze A, Fellner, Obletter N, Struhn R, Bautz W. Imaging and staging of avascular osteonecroses at the wrist and hand. Eur J Radiol 1997; 25:92-103.
11. Cerezal L, Abascal F, Canga A, et al. Usefulness of gadolinium-enhanced MR imaging in the evaluation of the vascularity of scaphoid nonunions. AJR Am J Roentgenol 2000; 174:141-149.

12. Ganapathi M, Savage R, Jones AR. MRI assessment of the proximal pole of the scaphoid after internal fixation with a titanium alloy Herbert screw. J Hand Surg [Br] 2001; 26:326-329.

13. Sakuma M, Nakamura R, Imaeda T. Analysis of proximal fragment sclerosis and surgical outcome of scaphoid nonunion by magnetic resonance imaging. J Hand Surg [Br] 1995; 20:201-205.

14. Beutel FK, Wilhelm K. Dependence of pattern of avascular necrosis on location and fracture line in scaphoid nonunion. Handchir Mikrochir Plast 1999; 31:207-211.

15. Günal I, Ozcelik A, Gokturk E, Ada S, Mehmet D. Correlation of magnetic resonance imaging and intraoperative punctuate bleeding to assess the vascularity of scaphoid nonunion. Arch Orthop Trauma 1999; 119:285-287. 